\title{
DETERMINATION OF THE RATE OF DISAPPEARANCE OF MUS- TARD GAS AND MUSTARD INTERMEDIATES IN CORNEAL TISSUE ${ }^{1}$
}

\author{
BY V. EVERETT KINSEY AND W. MORTON GRANT \\ (From Howe Laboratory of Ophthalmology, Harvard Medical School, Boston)
}

(Received for publication April 10, 1946)

Prior to World War II, the time of persistence of mustard gas (bis $\beta$-chloroethyl) sulfide (hereafter referred to as $\mathrm{H}$ ), in tissues had not adequately been determined. Although it was known that in aqueous environments $\mathrm{H}$ is rapidly hydrolysed to innocuous products, it seemed possible that this substance, because of its high lipoid solubility, might persist for much longer periods in the lipoid fractions of tissues. This possibility required investigation because of its bearing upon the potential efficacy of methods of decontamination, or treatment based upon the introduction into tissues of substances capable of reacting with and detoxifying $\mathrm{H}$. The results of numerous investigations carried out during the war years have established that $\mathrm{H}$, as such, rapidly disappears from the skin and eye. The present paper, based on work carried out in 1942, presents the results of one of the quantitative chemical investigations bearing on this point. Similar conclusions may be derived from the results of other work, notably that of Henriques, Mortiz, Breyfogle and Patterson (1941 to 1944) (1) on the skin, and that of Snell $(1942,1943)(2)$ on the cornea.

It is the purpose of this paper to report the results of an investigation designed to measure the rate of disappearance of $\mathrm{H}$ in tissue (cornea), and to correlate the quantity of $\mathrm{H}$ reacting with the degree of one of the effects produced by $\mathrm{H}$ on corneal tissue, viz., inhibition of turgescence which results normally when corneas are immersed in water. The latter effect was chosen for quantitating the effect, since previous studies by Cogan and the authors had shown that exposure to $H$ gave rise to reproducible changes in the degree of swelling.

1 The work described in this paper was done under a contract, recommended by the Committee on Medical Research, between the Office of Scientific Research and Development and Harvard University.

\section{METHODS}

Trephine buttons (70 mgm.) were cut from beef corneas and immersed for 10 minutes in liquid $H$ which was kept at a temperature of $12^{\circ} \mathrm{C}$. to minimize the chemical reaction of $\mathrm{H}$ with the cornea during the immersion period. The corneal buttons were then quickly rinsed in 3 washes of purified kerosene (hereafter called PK), and 1 wash of petroleum ether. Following this they were either extracted immediately for 2 hours in a mixture of 80 per cent cyclohexane and 20 per cent $\mathrm{PK}$ at $0^{\circ} \mathrm{C}$., or else were placed in empty stoppered bottles for varying lengths of time, and maintained at $23^{\circ} \mathrm{C}$. or $37^{\circ} \mathrm{C}$. to permit the $\mathrm{H}$ to react before extraction of any unchanged H.

Analyses of these extracts for $H$ were performed by the method of Kinsey and Grant (3).

Following the extraction period, the corneal buttons were allowed to swell in distilled water for 18 hours and were then weighed. The amount of swelling was calculated on the basis of 100 per cent. as the initial weight. Cyclohexane and PK used in the extraction process were found to have no effect on subsequent corneal swelling in water. Fifty control corneal buttons swelled an average of $\mathbf{5 7 0}$ per cent.

\section{RESULTS}

The curves of Figure 1A show the amount of unreacted $\mathrm{H}$ remaining in the corneal pieces after standing for various lengths of time at $23^{\circ} \mathrm{C}$. and $37^{\circ} \mathrm{C}$. following the 10 minute exposure period. It will be seen that half of the $\mathrm{H}$ no longer can be recovered after 13 and 3 minutes for these temperatures, respectively. These half-lives correspond almost exactly to those found for $\mathrm{H}$ in $\mathbf{0 . 6 0}$ per cent $\mathrm{NaCl}$ solution at the same temperatures. There was considerable variation in the amount of recoverable $\mathrm{H}$ in individual corneal pieces, which necessitated the use of relatively large number of pieces for each point composing the curves.

Figure 1B shows the amount of turgescence observed on these same corneal pieces. It is apparent from the figure that the observed effect on the cornea, as measured by the degree of inhibition 


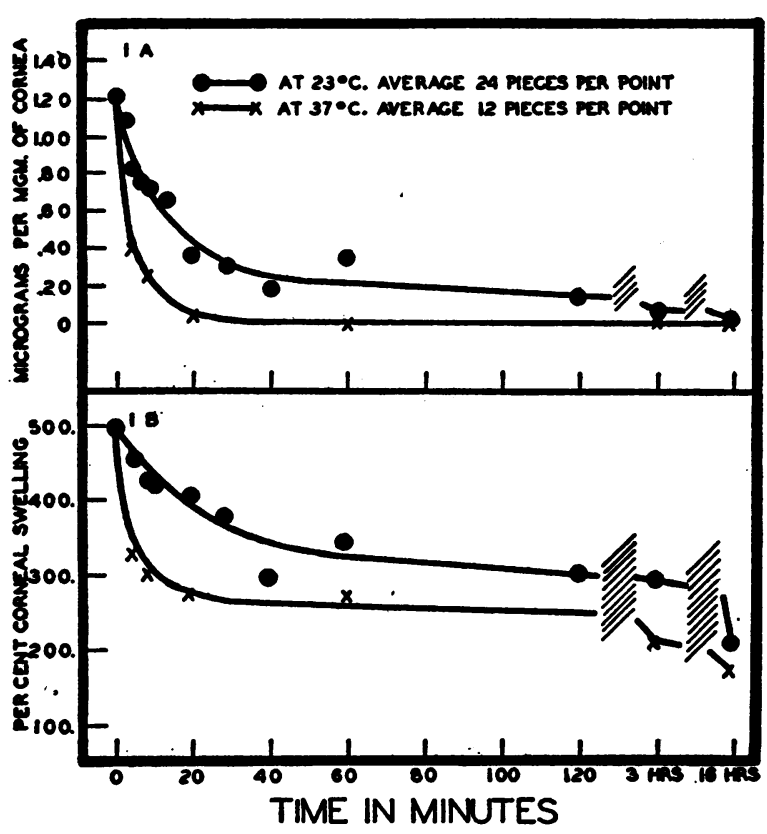

Fig. 1A. The Amount of Unreacted H Remaining in Corneal Pieces after Standing for Various Lengths of TIME AT $23^{\circ} \mathrm{C}$. or $37^{\circ} \mathrm{C}$.

Fig. 1B. The Amount of Turgescence Found in Corneal Pieces after Standing Various lengths of Time Following a 10-Minute Exposure to H

of turgescence, tends to parallel the disappearance of the $\mathrm{H}$ at the 2 temperatures. This relationship of effect produced for a given amount of $\mathrm{H}$ reacted, at the 2 temperatures investigated, may be more clearly seen from Figure 2. There the amount of $\mathrm{H}$ left in the cornea is plotted against effect, expressed as per cent inhibition of turgescence, where

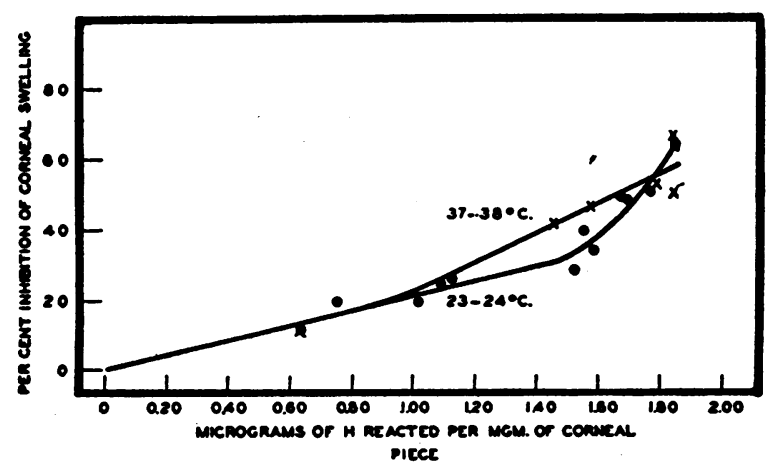

Fig. 2. The Relation Between the Effect Produced on Inhibition of Corneal Turgescence and the Quantity of H Which had Reacted within the CORNEA
100 per cent inhibition represents absence of swelling capacity, and 0 per cent inhibition represents swelling of normal unexposed corneas (570 per cent). It will be seen from the line composed of filled circles, which shows the data from the $23^{\circ} \mathrm{C}$. experiments, that the effect up to approximately $1.4 \gamma$ of $\mathrm{H}$ per mgm. of cornea, varies directly with the amount of $\mathrm{H}$ which has reacted. Above this amount it is evident that the effect no longer is directly proportional to the amount of $\mathrm{H}$ reacted, but increases faster than $\mathrm{H}$ disappears. Moreover, the effect from a given amount of reacted $\mathrm{H}$ appears to be somewhat greater at $37^{\circ} \mathrm{C}$. (crosses,

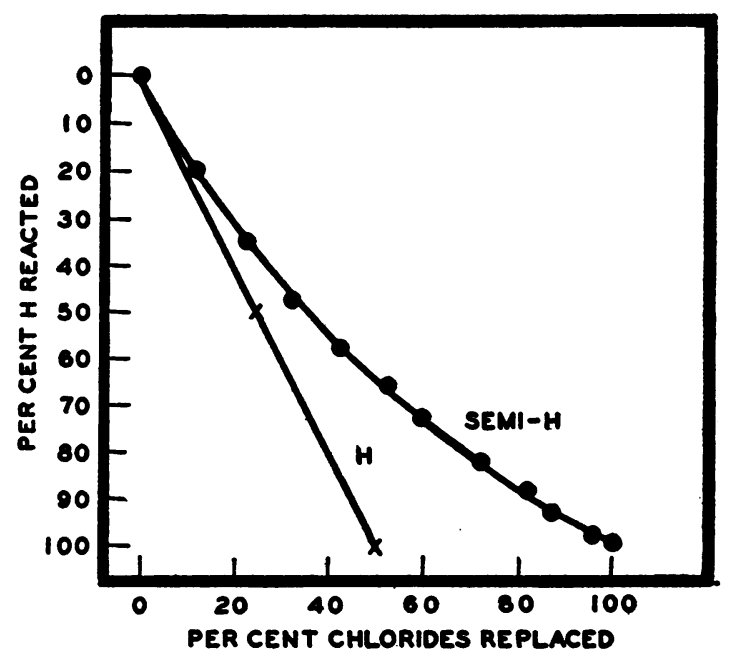

Fig. 3. The Relation Between the Percentage of H Reacting and the Percentage of Available Chlorides in the $\mathrm{H}$ or Semi-H Which Have BEEN REPLACED

Figure 3) than at room temperature. Both the changing slope of the $23^{\circ} \mathrm{C}$. line, and the apparently more uniform effect produced at $37^{\circ} \mathrm{C}$. are explicable on the assumption that 1 or more additional substances are formed which also contribute to the inhibition of turgescence. From studies being pursued simultaneously, it was found that chloroethyl-hydroxy-ethyl sulfide, hereafter referred to as Semi-H, is produced by the degradation of $\mathrm{H}$ in aqueous solution. It seemed probable that the presence of Semi-H, which was not determined in $\mathrm{H}$ analyses, might account for the linearity of the $23^{\circ} \mathrm{C}$. curve, as well as the greater observed effect per unit of reacted $\mathrm{H}$ at $37^{\circ} \mathrm{C}$. To test this hypothesis it seemed necessary to establish whether the effect produced in the corneal 
tissue more closely paralleled the replacement of both chlorides of $\mathrm{H}$. It is apparent that if this idea were substantiated it would be of practical importance to know the overall rate of reaction of $\mathrm{H}$ as well as its reactive products.

The rate of replacement of the first chloride is given directly by the measurement of the rate of disappearance of $\mathrm{H}$. An approximation of the rate of replacement of the second chloride can be obtained from the rate of breakdown of the chief analogue of $\mathrm{H}$ in which 1 chloride has been replaced, i.e., Semi-H.

The rate of breakdown of this compound was determined experimentally in 0.60 per cent saline and compared with that for $H$. From these data the proportion of both the first and second chlorides which would be replaced when any given fraction of $\mathrm{H}$ had hydrolyzed, was calculated from the equation representing the consecutive reactions which occur when $H$ hydrolyzes in 0.60 per cent saline solution. This was confirmed experimentally using pure Semi-H, and the temperature coefficients for the rate of replacement of the first and second chlorides were also found to be essentially the same. It follows that the total amount of replaceable chloride which has been available for any given fraction of $\mathrm{H}$ hydrolyzed is independent of temperature.

Hence, the data from Figure 3 may be used to determine the total number of chlorides which have been available for replacement from both $\mathrm{H}$ and Semi-H.

The amounts of $\mathrm{H}$ as taken from Figure 2 were now expressed on a percentage basis, whereby $1.84 \gamma$ of $\mathrm{H}$ per mgm. of córnea represented 100 per cent of the $\mathrm{H}$ reacted. The proportion of the total chlorides replaced for each percentage of $\mathrm{H}$ reacted was read off Figure 3 (filled circles). These values were now used as the abscissa of Figure 4 and plotted against effect produced (per cent inhibition). Thus, Figure 4 shows the effect produced on the cornea as a function of the proportion of total replaceable chlorides. It will be seen that the effect is closely proportional to the number of chlorides available for replacement from both $\mathrm{H}$ and Semi-H. This is in contrast to the poor proportionality found when $\mathrm{H}$ alone was considered to be effective (Figure 2). From the fact that this relationship holds equally well whether the measurements were made at $23^{\circ} \mathrm{C}$. (filled circles) or $37^{\circ} \mathrm{C}$. (crosses), it is apparent that the relationship is independent of rate of reaction.

Since it appears from the foregoing that $\mathrm{H}$ hydrolysis intermediates are capable of producing the same effect as the original $\mathrm{H}$ on the cornea (inhibition of swelling), it is necessary to know how long these active substances may be present in the cornea. This information can be obtained from Figure 3, where it will be seen that in 0.60 per cent saline (as found in the cornea) the "overall" half-life of $\mathrm{H}$ and its monochloro intermediate, Semi- $\mathrm{H}$, is about 1.4 times longer than the half-life of $\mathrm{H}$ itself. Applying this factor to the

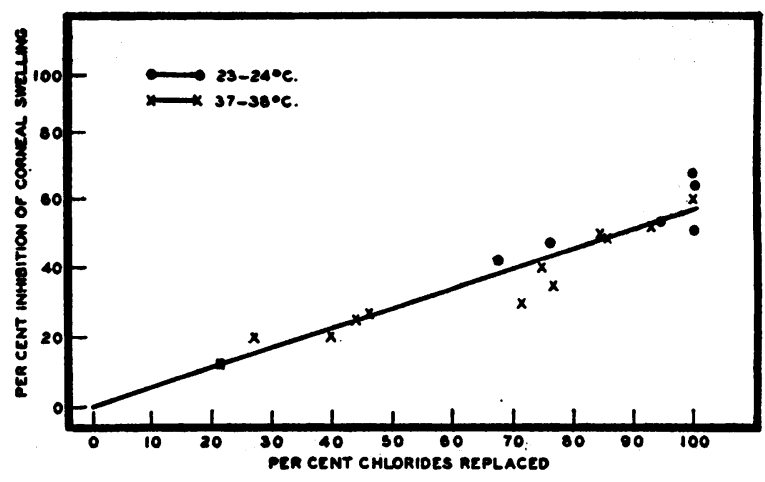

Fig. 4. The Relation of the Inhibition of Corneal Turgescence and the Amount of $\mathrm{H}$ Plus $\mathrm{H}$ Hydrolysis Products Which Have Reacted at $23^{\circ} \mathrm{C}$. OR $37^{\circ} \mathrm{C}$.

experimental measurements of the rate of $\mathrm{H}$ disappearance in the cornea, it is apparent that where the half-life at $23^{\circ} \mathrm{C}$. was 13 minutes, the overall half-life was $181 / 2$ minutes. At $37^{\circ} \mathrm{C}$. the halflife of $\mathrm{H}$ was 3 minutes, and the overall half-life was 4.2 minutes. It will be recalled that the "overall" half-life also gives an expression of the time required for production of half the observed inhibition of corneal swelling.

\section{DISCUSSION}

Although corneal turgescence was used as a convenient measure to quantitate the effect produced, the dose of $\mathrm{H}$ required to produce a significant effect on inhibition is far greater than that which gives rise to severe ocular lesions. It should 
not be implied, therefore, that inhibition of corneal turgescence is to be considered as a primary sign of $\mathrm{H}$ poisoning in the eye.

These experiments indicate that $\mathrm{H}$ and its labile hydrolysis intermediate, presumably Semi$\mathrm{H}$, exist as such, in corneal tissue for a remarkably short time at body temperature, and that the reaction which occurs during this period results in at least one effect on the tissue. No evidence was found to indicate that the hydrolysis rate in the cornea is essentially different from that in pure aqueous solutions. Thus, in this tissue, at least, no preservation of $\mathrm{H}$ in the lipoid phase was evident.

In view of the frequent attempts to institute therapy against $\mathrm{H}$ poisoning by introducing substances into tissue which are designed to react with $\mathrm{H}$ or its toxic degradation products, it is important to point out that the results of the present investigation would indicate that such treatment would have to be carried out within less than 3 to 5 minutes to be of practical value.

\section{SUMMARY}

1. The rate of disappearance of $\mathrm{H}$ in corneas in vitro at $23^{\circ} \mathrm{C}$. and $37^{\circ} \mathrm{C}$. was determined by analysis of cyclohexane-kerosene extracts. For these 2 temperatures, the half-life of $\mathrm{H}$ was found to be approximately 13 and 3 minutes, and the over-all half-life of $\mathrm{H}$ and its monochloro hydrolysis product was $181 / 2$ and 4 minutes, all respectively.

2. The effect on the corneal tissue was measured by subsequently determining inhibition of swelling of the corneal pieces when placed in water. It was found that the effect was proportional to the number of chlorides of both $\mathrm{H}$ and $\mathrm{H}$ hydrolysis intermediates available for replacement.

3. The findings indicate that therapeutic agents designed to react with $\mathrm{H}$ within tissues would be without benefit unless used within 3 to 5 minutes after contact with the vesicant.

\section{BIBLIOGRAPHY}

1. Unpublished data obtained in the United States.

2. Unpublished data obtained in the United States.

3. Kinsey, V. E., and Grant, W. M., Ind. \& Eng. Chem., Anal. Ed. (1946). 